\title{
Communication System Design and Implementation for Pico-Satellite and Earth Stations
}

\author{
J. J. Paternina-Anaya, MsC. ${ }^{1}$, J. E. Salamanca-Céspedes, MsC. ${ }^{1}$, and M. A. Ávila-Angulo, MsC. ${ }^{1}$ \\ ${ }^{1}$ Universidad Distrital Francisco José de Caldas, Colombia, jpaternina@udistrital.edu.co, jsalamanca@udistrital.ediu.co, \\ mavila@udistrital.edu.co
}

\begin{abstract}
This paper will describe all the hardware and software necessary to implement a pico-satellite network and 4 earth stations. The mission of the CubeSat, Colombia 1, Distrital University's research project, consists of taking ECG signals at an earth station and send it via pico-satellite to another. It was initially described as a network in the laboratory as wire is then implemented as happened to a wireless network.
\end{abstract}

Keywords- Communications, Networks, Pico-Satellite, Protocol AX.25, ECG, Signals.

Digital Object Identifier (DOI): http://dx.doi.org/10.18687/LACCEI2015.1.1.126

ISBN: 13 978-0-9822896-8-6

ISSN: 2414-6668

13 $^{\text {th }}$ LACCEI Annual International Conference: “Engineering Education Facing the Grand Challenges, What Are We Doing?” July 29-31, 2015, Santo Domingo, Dominican Republic 


\title{
Communication System Design and Implementation for Pico-Satellite and Earth Stations
}

\author{
J. J. Paternina-Anaya, MsC, J. E. Salamanca-Céspedes, MsC, and M. A. Ávila-Angulo, MsC, \\ Universidad Distrital Francisco José de Caldas, Colombia, jpaternina@udistrital.edu.co, \\ jsalamanca@udistrital.ediu.co, mavila@udistrital.edu.co
}

\begin{abstract}
This paper will describe all the hardware and software necessary to implement a pico-satellite network and 4 earth stations. The mission of the CubeSat, Colombia 1, Distrital University's research project, consists of taking ECG signals at an earth station and send it via pico-satellite to another. It was initially described as a network in the laboratory as wire is then implemented as happened to a wireless network.
\end{abstract}

Index Terms-Communications, Networks, Pico-Satellite, Protocol AX.25, ECG, Signals.

\section{INTRODUCCIÓN}

$\mathrm{L}$ A Universidad Distrital Francisco José de Caldas y el grupo de investigación en Telemedicina - GITEM se ha encargado del proceso de investigación, diseño y desarrollo de este proyecto. Las comunicaciones entre el pico satélite Colombia 1 y las estaciones terrenas se realizan en banda de radio aficionado y la transmisión de datos para este tipo de redes es realizada en el protocolo AX.25. El sistema de comunicaciones además de manejar este protocolo debe incluir un protocolo para intercambio de información con la tarjeta central, sistema de despliegue de antena en vuelo, sistema de radio frecuencia e interfaz entre la parte digital y el sistema de radio frecuencia. En el nivel físico con que se implementa AX.25 es NRZI [1], y el hardware con que se implementó se hizo en base al microcontrolador MSP430F1612 de "Texas Instruments", con la tarjeta principal se utilizó un protocolo de hardware basado en señales de modem que más adelante se describirán. La implementación del TNC (Controlador de Nodo Terminal) tanto del pico satélite como de las estaciones terrenas se realizó basado en el modem FX614, el cual realiza modulación FSK BELL 202.

\section{ANTECEDENTES}

A. Proyecto Pico satélite Cal Poly CP1 de la Universidad Politécnica de California [2].-

Es la primera misión de pico satélite de esta Universidad, la cual consistió en probar distintos dispositivo como micro- rocket, magneto-torquer, sistemas micro-electro-mecánico (MEM) y una gran variedad de sensores. Las comunicaciones de RF en esta misión se implementaron con radio de la marca ALINCO en la banda de radio aficionado de $70 \mathrm{mts}$, debido a la poca cantidad de datos para enviar la implementación en velocidad fue de solo $60 \mathrm{bps}$, en una combinación de DTMF y código MORSE. En esta misión solo se requiere una conexión punto a punto entre el pico satélite y la estación terrena.

\section{B. Proyecto AAU-CubeSat de la Universidad de Alborg de Dinamarca [3].-}

La misión de este proyecto era tomar fotografía de la superficie de la tierra y transmitirla a una estación terrena (enlace punto a punto). Debido al gran volumen de información a transmitir fue necesario implementar la velocidad de transmisión a 9600 bps en el enlace AX.25 implementándose, la modulación en banda base con el chip MODEM MX909[4], el cual implementa modulación de fase mínima Gaussiana (GMSK).

\section{Proyecto Pico satélite Cal Poly CP2 de la Universidad Politécnica de California [5]. -}

En este proyecto se concibe implementar AX.25 a $1200 \mathrm{bps}$ con el transceiver CC1000, pero por limitaciones técnicas solo se logra comunicaciones a $600 \mathrm{bps}$, lo cual no implica gran inconveniente ya que el volumen de transmisión a transmitir solo era de 256 bytes máximo.

\section{DESCRIPCIÓN DE AX.25}

Este protocolo es utilizado en la transmisión de datos en redes de radio aficionados, y es también utilizado en enlaces de pico satélites y estaciones terrenas. AX.25 posee 3 tipos de tramas: Tramas de supervisor, tramas no numeradas y tramas de información [6]. Estas tramas son identificadas en el campo de control que existen en cada trama y que se indican en la Tabla I. N(R), indica la trama de información que esta por recibirse y $\mathrm{N}(\mathrm{S})$, indica la trama de información que se está transmitiendo.

El campo de control puede tener 8 o 16 bits dependiendo si AX.25 es módulo 8 o módulo 16. Si es módulo $16 \mathrm{~N}(\mathrm{R})$ y $\mathrm{N}(\mathrm{S})$ son de 7 bits en vez de 3 bits como aparece en la Tabla I, 
TABLA I

TRAMAS UTILIZADAS EN AX.25

\begin{tabular}{l|ll|l|lll}
\hline \hline $\begin{array}{l}\text { Tipo de } \\
\text { Trama }\end{array}$ & $\begin{array}{l}7 \\
5\end{array}$ & 6 & 4 & 3 & 2 & 1 \\
\hline Información & $\mathrm{N}(\mathrm{R})$ & & $\mathrm{P}$ & & $\mathrm{N}(\mathrm{s})$ & $\mathrm{i}$ \\
& & & 0 & & \\
\hline Supervisor & $\mathrm{N}(\mathrm{R})$ & & $\mathrm{P} / \mathrm{F}$ & $\mathrm{S}$ & $\mathrm{S}$ & 0 \\
& & & & 1 & & \\
\hline No & $\mathrm{M}$ & $\mathrm{M}$ & $\mathrm{P} / \mathrm{F}$ & $\mathrm{M}$ & $\mathrm{M}$ & 1 \\
Numerada & $\mathrm{M}$ & & & 1 & & \\
\hline \hline
\end{tabular}

para el módulo 8. El módulo 8 es el implementado en este proyecto.

La programación del tipo de modulo se hace a través de la primera trama no numerada enviada en el enlace.

En la Tabla II se observa los campos de una trama de información AX.25, el campo de información puede ser de 112 o 224 bits, en este proyecto se realizó a 112 bits: 7 bytes para la dirección destino y 7 bytes para la dirección origen. El campo PID el cual identifica el protocolo encapsulado dentro del campo de información, cuando no existe tal encapsulamiento este campo se coloca en F0 hexadecimal.

En la Tabla III aparecen las tramas de supervisor utilizadas por AX.25 y las cuales fueron implementadas en este proyecto para módulo 8.

Cuando hay un enlace establecido la trama RR se utiliza para indicar que está listo para recibir tramas y en el campo $\mathrm{N}(\mathrm{R})$, la trama que esta por recibir, validando de esta forma las tramas precedentes y que no se habían validado. La trama "Reject" solicita retransmisión de todas las tramas desde una trama en particular. La trama "Selective Reject", solicita retransmisión de una única trama. En la Tabla IV aparecen las tramas no numeradas.

Cuando el master desea establecer un enlace envía SABME (Set Asyn Balanced Mode Extended) para módulo 16 o SABM (Set Asyn Balanced Mode), para módulo 8. La estación o esclavo que acepta el comando correspondiente responde con UA (unnumbered acknowledge).

Cuando no hay información que intercambiar el pico satélite

TABLA II

TRAMAS DE INFORMACIÓN AX.25

\begin{tabular}{c|c|c|c|c|c|c}
\hline \hline Flag & Address & Control & PID & Info & FCS & Flag \\
\hline $7 \mathrm{E}$ & $112 / 224 \mathrm{bit}$ & $8 / 16 \mathrm{bit}$ & $8 \mathrm{bit}$ & $\mathrm{N}^{\circ} 8$ & $16 \mathrm{bit}$ & $7 \mathrm{E}$ \\
\hline \hline
\end{tabular}

TABLA III

CAMPO DE CONTROL TRAMA DE SUPERVISOR

\begin{tabular}{l|ll|l|ll|l}
\hline \hline Tipo de Trama & 7 & 6 & 4 & 3 & 2 & $\begin{array}{l}1 \\
0\end{array}$ \\
\hline Receive Ready RR & 5 & $\mathrm{~N}(\mathrm{R})$ & $\mathrm{P} / \mathrm{F}$ & 0 & 0 & 0 \\
& & & & & 1 \\
\hline Receive Not Ready & $\mathrm{N}(\mathrm{R})$ & $\mathrm{P} / \mathrm{F}$ & 0 & 1 & 0 \\
& & & & & 1 \\
\hline Reject & $\mathrm{N}(\mathrm{R})$ & $\mathrm{P} / \mathrm{F}$ & 1 & 0 & 0 \\
& & & & & 1 \\
\hline Selective Reject & $\mathrm{N}(\mathrm{R})$ & $\mathrm{P} / \mathrm{F}$ & 1 & 1 & 0 \\
& & & & & & 1 \\
\hline \hline
\end{tabular}

TABLA IV

TRAMAS NO NUMERADAS

\begin{tabular}{|c|c|c|c|c|c|}
\hline Tramas & Tipo & 765 & 4 & 32 & 10 \\
\hline SABME & Cmd & 0111 & $P$ & 11 & 11 \\
\hline SABM & Cmd & 001 & $P$ & 11 & 11 \\
\hline DISC & Cmd & 010 & $P$ & 00 & 11 \\
\hline $\mathrm{DM}$ & Res & 000 & $\mathrm{~F}$ & 11 & 11 \\
\hline$\overline{\mathrm{UA}}$ & Res & 011 & $\bar{F}$ & 00 & 11 \\
\hline FRMR & Res & 100 & $\mathrm{~F}$ & 01 & 11 \\
\hline UI & Either & 000 & $\mathrm{P} / \mathrm{F}$ & 00 & 11 \\
\hline XID & Either & 101 & $\mathrm{P} / \mathrm{F}$ & 11 & 11 \\
\hline TEST & Either & 111 & $\mathrm{P} / \mathrm{F}$ & 00 & 11 \\
\hline
\end{tabular}

inicia una secuencia de desconexión con la trama no numerada DISC, a lo cual la estación terrena responde con UA.

\section{ARQUITECTURA DE LA RED AX.25 IMPLEMENTADA}

En la Fig. 1. aparece la red de un pico satélite y 4 estaciones terrenas propuesta. Cada estación corresponde a cada una de las siguientes ciudades: Bogotá, Tunja, Neiva y Florencia.

Para efecto de experimentación en el laboratorio se asignó arbitrariamente las direcciones indicada en la Fig. 1, donde la dirección 12345 corresponde al pico satélite.

El pico satélite hace de máster y las estaciones terrenas de esclavas. Se han previsto 2 situaciones para establecer un enlace: 1). Cuando el módulo de control de actitud del satélite informa al módulo de comunicaciones que esta sobre la región de las estaciones terrenas este inicia un "polling" a cada estación ya sea para el envío o recepción de información. 2). El pico satélite a través de la señal de "BEACON" envía la dirección con la estación con la cual desea establecer enlace y espera por su línea de recepción AX.25, la cual está permanentemente habilitada y dependiendo de si llego algo desencadena la secuencia de inicialización.

Para la implementación de AX.25 se desarrolló la red en estrella en forma alambrada tal como se indica en la Fig. 2.

El propósito de alambrar esta red fue probar la lógica de

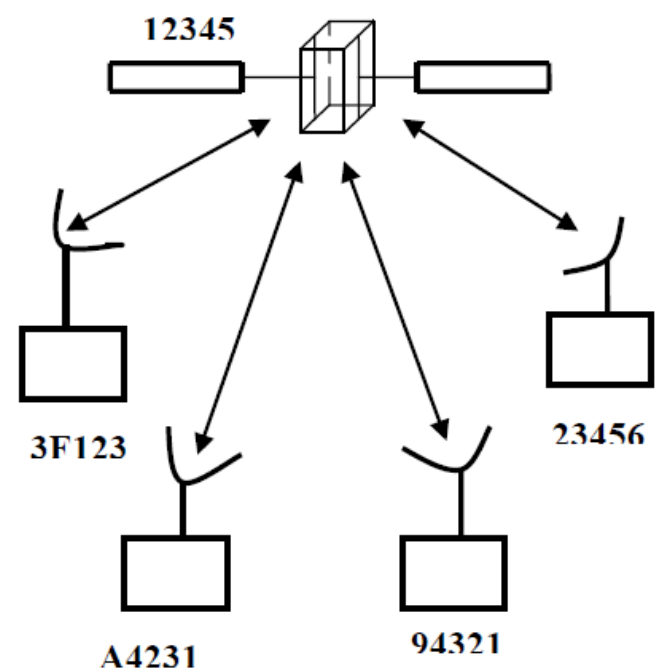

Fig. 1. Red en Estrella de un pico satélite y 4 estaciones terrenas

13 $^{\text {th }}$ LACCEI Annual International Conference: "Engineering Education Facing the Grand Challenges, What Are We Doing?" July 29-31, 2015, Santo Domingo, Dominican Republic 


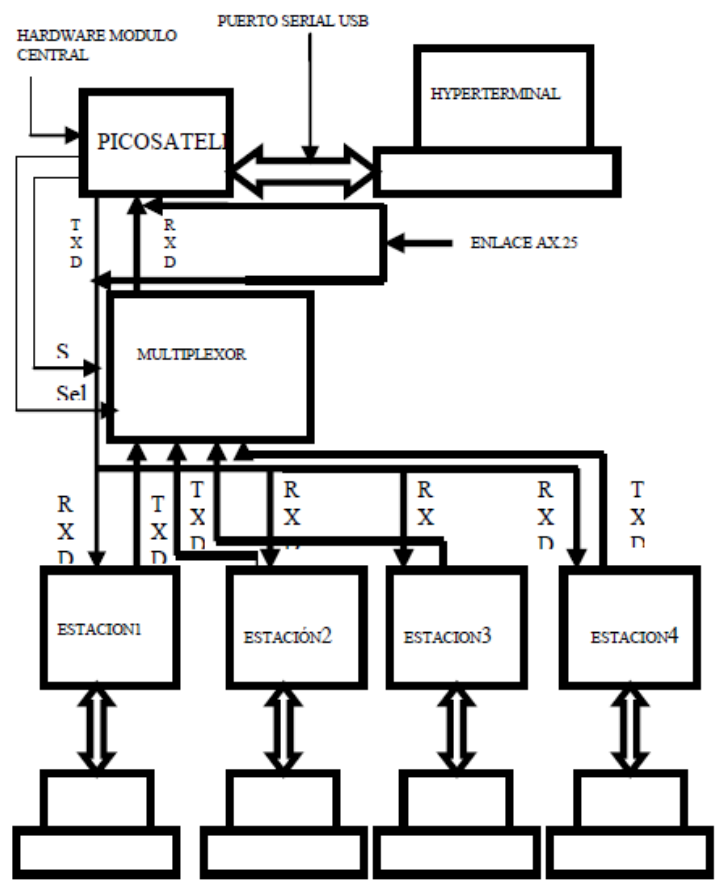

Fig. 2. Emulación alambrada de la red de la Fig. 1.

cada una de las rutinas que se iban desarrollando en laboratorio, a la tarjeta central del pico satélite, se conecta a través de un puerto USB a Hiperterminal, con el objeto de visualizar las tramas que se están intercambiando para poder ir ajustando el desarrollo final, lo que el pico satélite envía hacia el enlace también es enviado a Hiperteminal. Obviamente la opción de Hiperterminal cuando el pico satélite esta en órbita no va.

En la Fig. 2., se observa en el enlace AX.25 una línea de transmisión que llega a todas las estaciones terrenas en forma directa y una línea de recepción la cual es multiplexada desde cada uno de los transmit (TXD) de las estaciones terrenas. Cuando el pico satélite interroga a una estación terrena simultáneamente selecciona a través del multiplexor el "Transmit" de la estación correspondiente.

En la Fig. 3., se observa tramas SABM, enviadas desde el pico satélite a las estaciones terrenas. Nótese que el pico satélite interroga las estaciones con direcciones: 94321, A4231, 3F123 y 94321 pero ninguna responde.

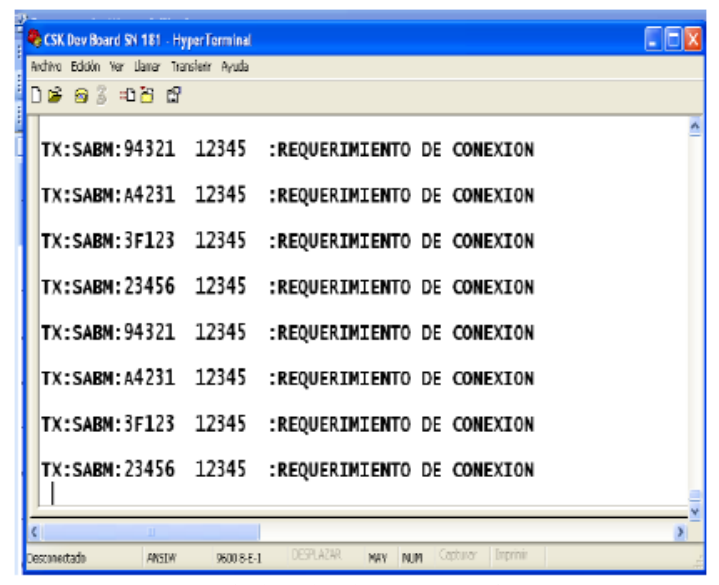

Fig. 3. Tramas SABM enviadas al enlace AX.25 sin respuesta.

\begin{tabular}{|c|c|}
\hline COCK Der Faard SN 181 - Hype Teminal & E]可 \\
\hline \multicolumn{2}{|l|}{ 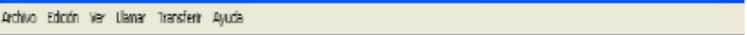 } \\
\hline \multicolumn{2}{|l|}{ 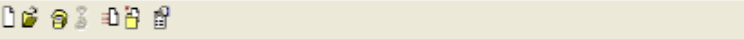 } \\
\hline TX:SABM:3F123 12345 :REQUERIMIENTO DE CONEXION & $\hat{=}$ \\
\hline TX:SABM:23456 12345 :REQUERIMIENTO DE CONEXION & \\
\hline RX:UA:12345 23456 :CONEXION ACEPTADA & \\
\hline TX:RR:0:23456 $12345:$ & \\
\hline RX:RR:0:12345 $23456 \quad:$ & \\
\hline TX:DIsC:23456 12345 :SOLICITUD DE DESCONEXION & \\
\hline RX:UA:12345 23456 :DESCONEXION ACEPTADA & \\
\hline TX:SABM:94321 12345 :REQUERTMIENTO DE CONEXION & \\
\hline TX:SABM:A4231 12345 :REQUERIMIENTO DE CONEXION & \\
\hline
\end{tabular}

Fig. 4. La estación 23456 acepta la conexión.

En la Fig. 4 se observa que la estación 23456, acepta el requerimiento de conexión, pero como no hay información que intercambiarse el pico satélite hace un requerimiento de desconexión el cual es aceptado por la estación.

En la Fig. 5 se observa que la estación 94321(estación 2) tiene información que intercambiar con el pico satélite, en la primera trama de información se observa con $\mathrm{N}(\mathrm{S})=$ 0 (enviando la trama cero) y $\mathrm{N}(\mathrm{R})=0$ (esperando la trama 0 ), y con información: 21UNIVERSIDAD DISTRITAL. Lo cual significa que ésta es la estación 2 y ésta trama debe reenviarla el pico satélite hacia la estación 1. Después de esto el pico satélite envía la trama de supervisor RR (Ready to receive), con $\mathrm{NR}(\mathrm{R})=1$, es decir, valida la trama cero recibida y está esperando la siguiente trama de información que es la 1.

Seguidamente la estación 94321(estación 2), envía la trama 1 con $\mathrm{N}(\mathrm{S})=1$ y $\mathrm{N}(\mathrm{R})=0$ e información: 21FACULTAD DE INGENIERIA (ver Fig. 5), el pico satélite la valida con una trama $R R$ con $\mathrm{N}(\mathrm{R})=2$, como la estación terrena no tiene más información que transmitir el pico satélite inicia una secuencia de desconexión que es aceptada por la estación terrena (ver Fig. 5). Ahora el pico satélite tiene información para transmitir a la estación 23456(estación 1) en la Fig. 6 se observa cómo se envía esta información desde el pico satélite.

En las estaciones terrenas se desarrolló un hardware y software para soportar el "dialogo" AX.25 descrito hasta aquí

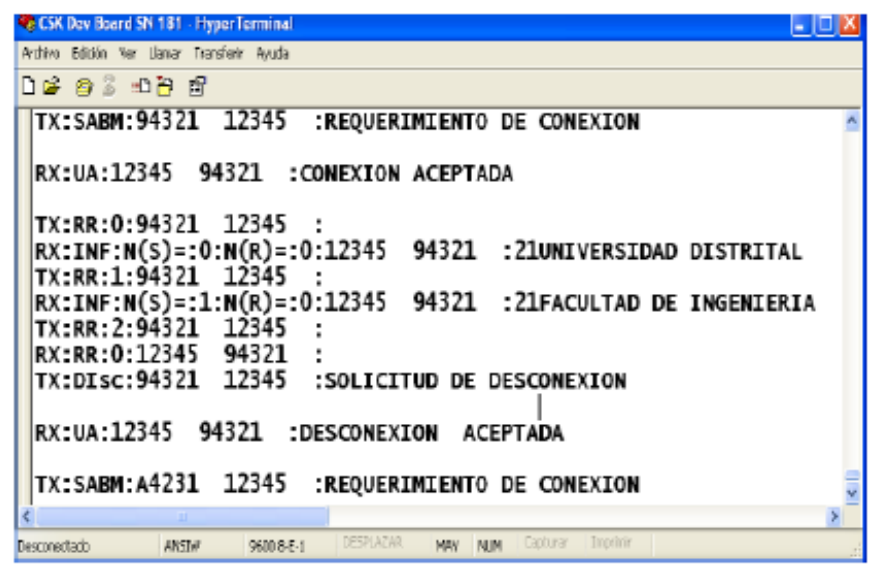

Fig. 5. La estación 94321 con información al pico satélite.

13 "' LACCEI Annual International Conference: "Engineering Education Facing the Grand Challenges, What Are We Doing?" July 29-31, 2015, Santo Domingo, Dominican Republic 


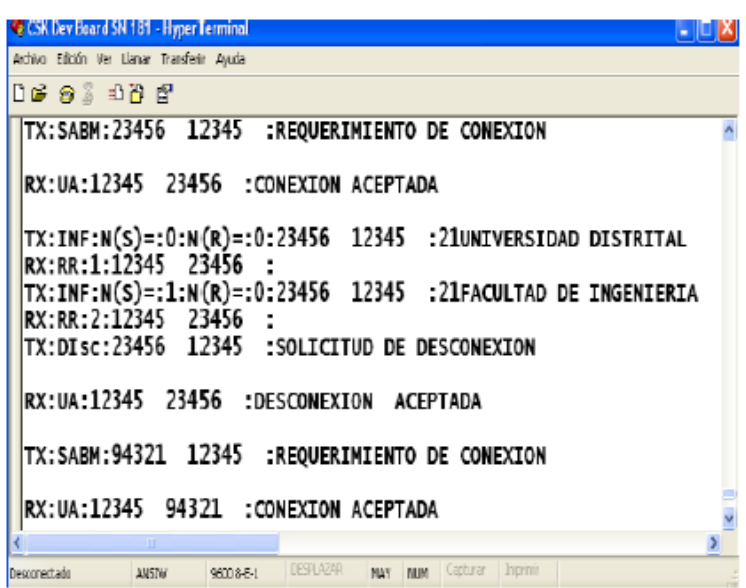

Fig. 6. Descargando información del pico satélite a la estación 23456 (estación 1).

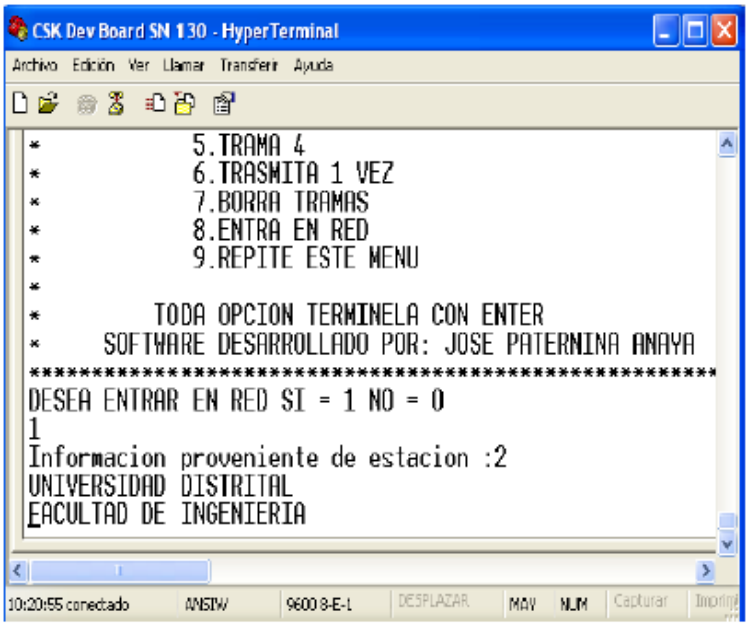

Fig. 7. Descargando información a la estación 1.

en el pico satélite, esto permite visualizar la información que llega indicando la estación de donde proviene (ver Fig. 2 y Fig. 7)

La aplicación desarrollada en las estaciones terrenas también permite editar:

a. Estación destino

b. Hasta 4 tramas de información

c. Posibilidad de transmitir una vez las tramas editadas

d. Configuración para que la estación entre en red

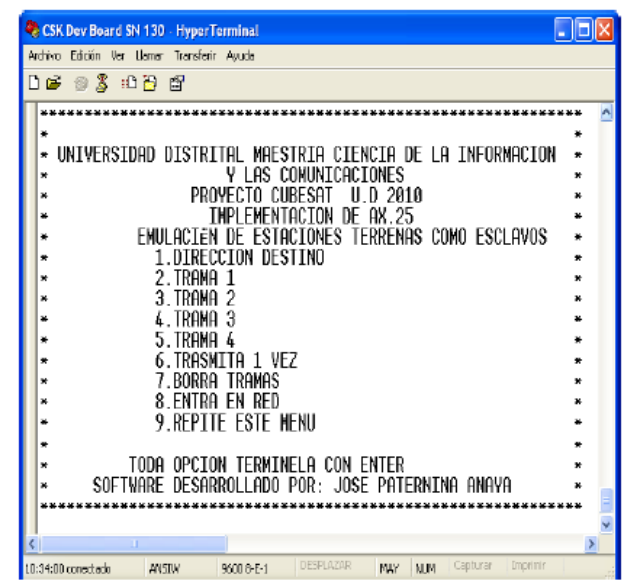

Fig. 8. Presentación en híper terminal de aplicación para estaciones terrenas. e. Borrar todas las tramas editadas

Esto se ilustra en la Fig. 8.

\section{IMPLEMENTACIÓN DEL TNC PARA EL PICO SATÉLITE Y LAS ESTACIONES TERRENAS}

Toda la información digital que viaja en la red alambrada descrita en la sesión anterior es necesario modularla para la parte de transmisión y demodularla para la parte de recepción esto se hace en AFSK, para colocarla en la entrada de audio de los transmisores en banda de radioaficionado.

En la Fig. 9 se ilustra el diagrama de bloques para lograr la interfaz requerida.

Inicialmente el modulador AFSK se implementó con el modulador XR2206 [7] y el demodulador XR2211 [8] de EXAR, los cuales no presentaron los resultados esperados, en su lugar con mejor desempeño se utilizó el chip modem FX614 [9], el cual por presentar modulación coherente, como se muestra en la Fig. 10, presentó mejores resultados.

Una vez probada la modulación en banda base a 1200 BPS se procedió a la implementación en radio frecuencia, al cual se logró, para el pico satélite, con los radios comerciales YAETSU VX-3R, conectado la señal FSK a la entrada, tal como se ilustra en la Fig. 11. Este radio miniatura permite satisfacer los exigentes requerimientos de peso y volumen impuesto por el estándar CUBESAT (ver Fig. 12). En el pico satélite se acomoda en el módulo de comunicaciones el radio sin carcasa, una vez configuradas las correspondientes frecuencias de transmisión y recepción.

Cuando el pico satélite inicia una transmisión activa el PTT (push to talk), en los radios y envía la señal en base (BELL 202), para que esta sea modulada en RF.

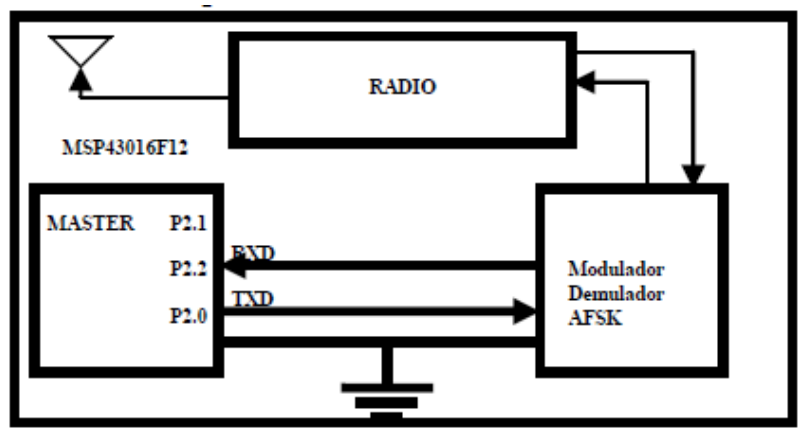

Fig. 9. Interfaz a radio frecuencia.

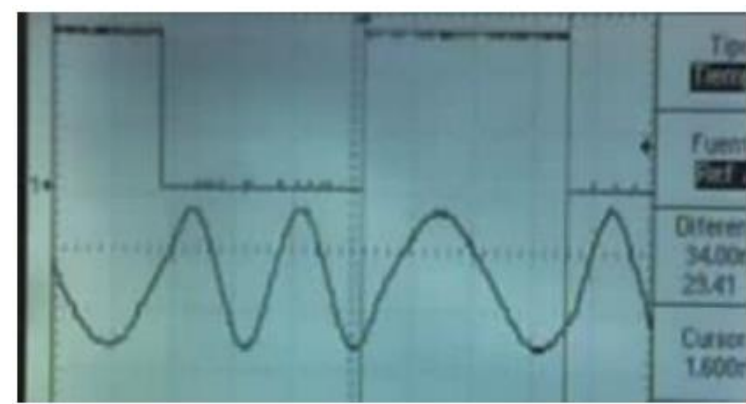

Fig. 10. Modulación con FX614 a 1200 BPS.

13 $^{\text {th }}$ LACCEI Annual International Conference: "Engineering Education Facing the Grand Challenges, What Are We Doing?" July 29-31, 2015, Santo Domingo, Dominican Republic 


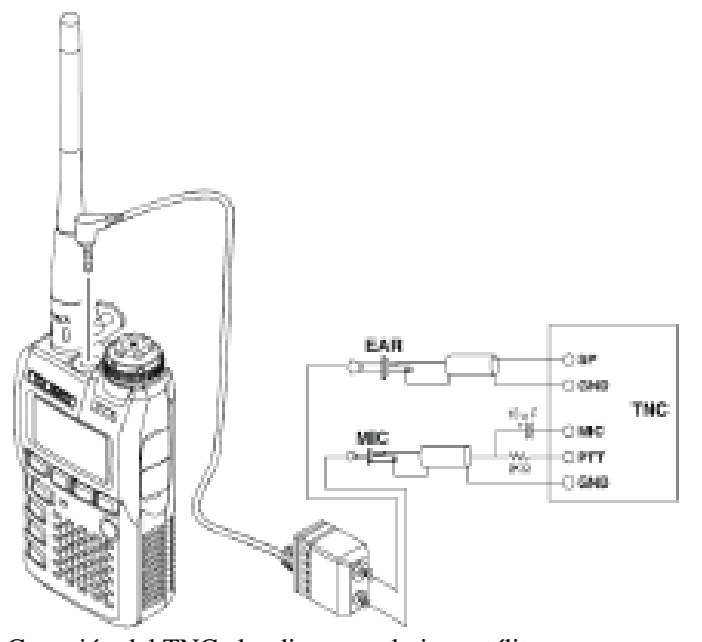

Fig. 11. Conexión del TNC al radio, para el pico satélite.

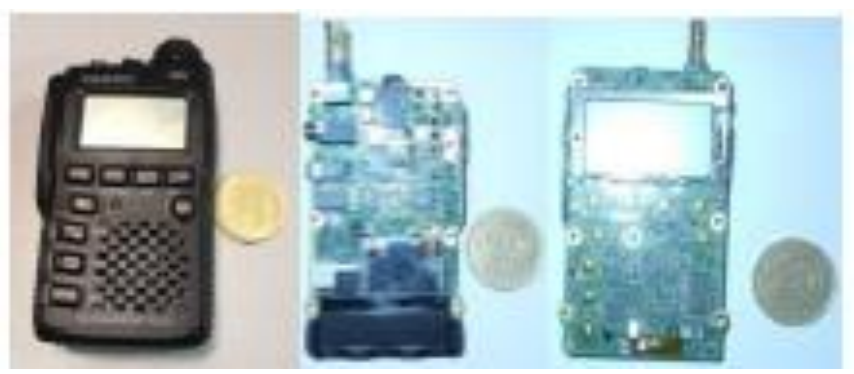

Fig. 12. Radio Yaetsu VX-3R con y sin carcasa.

\section{IMPLEMENTACIÓN DEL BEACON EN EL PICO SATÉLITE Y DETECCIÓN EN LAS ESTACIONES TERRENAS}

En el pico satélite, por las mismas líneas por donde se envían datos AX.25 se envía el BEACON. La trama de ésta contiene un identificador COL, datos de telemetría y datos de dirección con la cual el pico satélite desea establecer comunicaciones. Estos datos son codificados en MORSE y la forma como esto se hace se explica detalladamente en [10]. Esta señal BEACON permite la detección de nuestro satélite en cualquier parte del mundo donde se disponga de los decodificadores correspondiente.

En las estaciones terrenas, la señal de salida de audio del radio se utiliza como entrada a la tarjeta sonido de un P.C. como único requisito de hardware para decodificar la señal de BEACON. El software para este propósito se denomina CWGET, el cual se puede obtener en forma gratuita de http://cwget.softonic.com/. A través de un DLL éste software, establece un canal de comunicaciones con un programa escrito en plataforma visual NET, el cual se encarga de decodificar todas las tramas de BEACON que llegan a cada una de las estaciones terrenas, una vez este software detecta el BEACON con el encabezado COL, ordena automáticamente al TNC establecer un enlace AX.25 con el pico satélite.

En el pico satélite del proyecto CubeSat Colombia 1, la frecuencia para generar la señal de BEACON es la de $2200 \mathrm{~Hz}$ de los MODEM FX-614, lo cual se logra a través de un multiplexor análogo. Cuando se transmite el BEACON a la

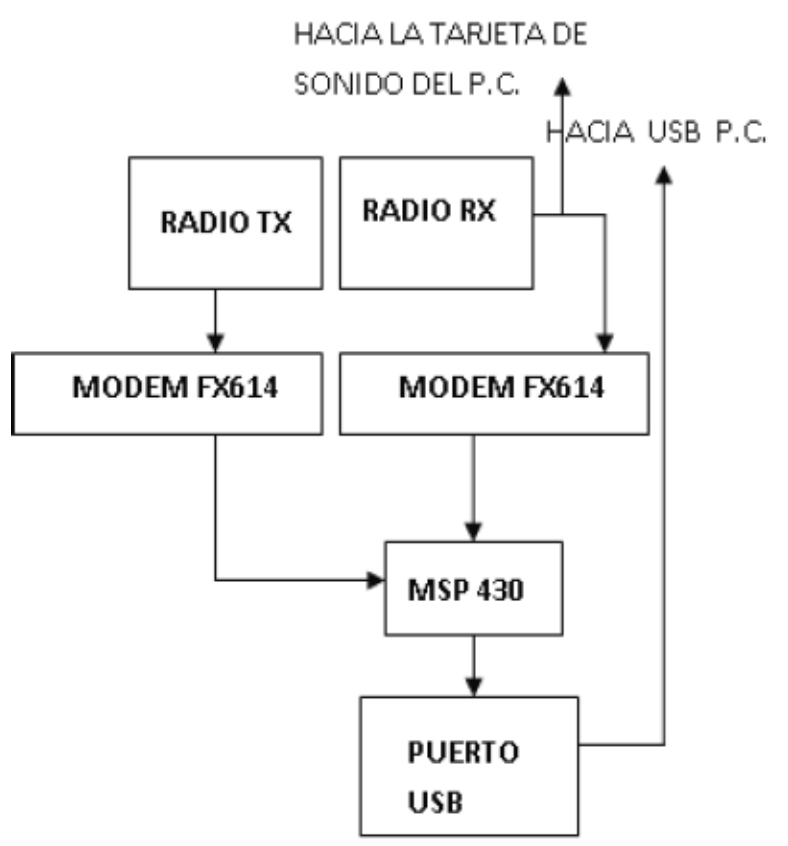

Fig. 13. Esquema de hardware para decodificar el BEACON y el enlace AX.25

salida del modem se deja fija la frecuencia de $2200 \mathrm{~Hz}$, logrando la transmisión morse prendiendo y apagando hacia el radio esta frecuencia.

Cuando se transmiten datos hacia el radio la salida del modem coloca las frecuencias de 1200 o $2200 \mathrm{~Hz}$ para unos y ceros respectivamente.

En el desarrollo de la red propuesta se hizo necesario además de monitorear BEACON, monitorear la información que viaja a través del enlace AX.25, el esquema de hardware para este propósito se muestra en la Fig. 13. Como se puede ver un radio monitorea la línea de recepción y el otro la línea de transmisión en la línea de recepción también se monitorea el BEACON. El software y el hardware desarrollado, para monitorear AX.25 se describen detalladamente en [11].

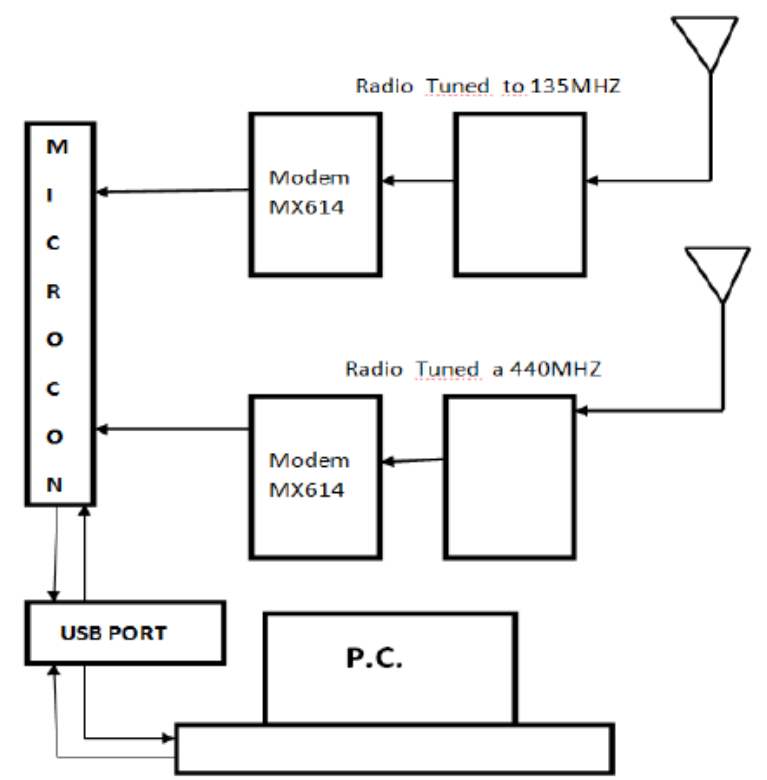

Fig. 14. Esquema usado para monitorear AX.25 inalámbricamente 


\section{CONCLUSIONES}

El desarrollo de esta red implico un desafío importante, porque constituye una de las misiones más complejas desarrollas en proyecto CubeSat alrededor del mundo. En el desarrollo de este sub-módulo, de comunicaciones fue necesario recorrer un arduo camino de frustraciones unas veces y éxitos otras. Fue necesario desarrollar 5 prototipos, cada uno con sus méritos y sus defectos pero de todas maneras fue un proceso de construcción permanente. Hoy todos los que hemos participado en este proceso tenemos una visión más integral de la ingeniería y de la forma como se debería trabajar en un proyecto interdisciplinario de investigación, a nivel científico nos ha quedado una base, incluyendo los restantes módulos para el desarrollo de tecnología aeroespacial.

Nos proponemos como objetivos de próximas misiones: Cuadriplicar la velocidad de transmisión de datos a 9600bps, implementar comunicaciones full dúplex con procesamiento paralelo en FPGA, Aportar en la discusión en la forma como se implementaría una constelación de pico satélite que la comunidad CubeSat desea implementar.

Todo este trabajo desarrollado nos está permitiendo la implementación de laboratorios para el aprendizaje de conocimientos básicos en ciencia y tecnología aeroespacial, todo esto en el ámbito nacional.

\section{REFERENCIAS}

[1] Paternina José, "Implementación de AX.25 en pico satélite Colombia 1", articulo presentado en el III congreso internacional de tecnología aeroespacial realizad lo en Bogotá en octubre 10 y 11 del 2010.

[2] Schaffner, Jake A, "The Electronic System Design, Analysis, Integration, and Construction of the Cal Poly State University CP1 CubeSat"

[3] Lars Alminde, Morten Bisgaard, DennisVinther, Tor Viscor and Kasper Z. Ostergaard, "Robustness of radio Link between AAU- Cubesat and Ground Station"

[4] MX-COM, INC Mixed Signal Data Transmission, "Practical GMSK Data Transmission, Aplication Note"

[5] Chris Noe, "Design and Implementation of the Communications Subsystem for Cal Poly CP2 Cubesat Project". Computer Engineering
Department California Polytechnic State University, San Luis Obispo Submitted: June, 2004

[6] Beech A. William, Douglas E. Nielsen, Jack, Taylor. "AX.25 Link Access Protocol for Amateur Packet Radio". Version: July 1998

[7] Exar Company: Data sheet XR2206

[8] Exar Company: Data Sheet XR2211.

[9] MX614. Datasheet del fabricante. MX.COM. Communication Semiconductors.

[10] Buitrago Diana,Valverde Nestor, Gaona Elvis. "Sistema de Telemetría del Picosatelite Colombia 1",articulo presentado en Ninth LACCEI Latin American and Caribbean Conference (LACCEI'2011), Engineering for a Smart Planet, Innovation, Information Technology and Computational Tools for Sustainable Development, August 3-5, 2011, Medellín, Colombia.

[11] Moncaleano Ariel, Paternina José, Gaona Elvis. "Como implementar un analizador de protocoloAX.25". articulo presentado en el IV congreso internacional de tecnología aeroespacial realizado en Bogotá, octubre 4y 5 de 2012.

José De Jesús Paternina Anaya, es ingeniero electrónico de la Universidad Distrital Francisco José de Caldas, Magister en Ciencias de la Información y las telecomunicaciones de la misma Universidad FJC, se encuentra trabajando como investigador del grupo de investigación GITEM de la Facultad de ingeniería de la Universidad Distrital en el tema de desarrollo de satélites. Docente asociado de tiempo completo adscrito a la facultad de ingeniería Proyecto curricular de ingeniería electrónica, con más de 40 años de experiencia en el campo docente y de ingeniería, investigador en desarrollo de picosatélites.

Jorge E. Salamanca Céspedes, es ingeniero electrónico de la Universidad Distrital Francisco José de Caldas, Especialista en telecomunicaciones Móviles y Magister en Ciencias de la Información y las telecomunicaciones de la misma Universidad, se encuentra trabajando como investigador del grupo de investigación GITEM de la Facultad de ingeniería de la Universidad Distrital FJC en el tema de desarrollo de satélites.

Docente asociado de tiempo completo adscrito a la facultad de ingeniería Proyecto curricular de ingeniería electrónica, con más de 25 años de experiencia en el campo docente y de ingeniería, investigador en desarrollo de picosatélites.

Miguel A. Ávila Angulo, es ingeniero Catastral y Geodesta de la Universidad Distrital Francisco José de Caldas, Magister en Ciencias de la Información y las telecomunicaciones de la misma Universidad FJC, se encuentra trabajando como investigador del grupo de investigación GITEM de la Facultad de ingeniería de la Universidad Distrital en el tema de desarrollo de satélites. Docente asociado de tiempo completo adscrito a la facultad de ingeniería Proyecto curricular de ingeniería electrónica, con más de 20 años de experiencia en el campo docente y de ingeniería, investigador en desarrollo de picosatélites. 